\title{
Die Reflektivität von Rubidium- und Cäsium-Suboxiden
}

\author{
G. Metsch, W. Bauhofer und A. Simon \\ Max-Planck-Institut für Festkörperforschung, Stuttgart \\ Z. Naturforsch. 40 a, $303-312$ (1985); received January 24, 1985 \\ The Reflectivity of $R b$ and $C s$ Suboxides
}

The reflectivity of single crystals of the alkali metal suboxides $\mathrm{Cs}_{7} \mathrm{O}, \mathrm{Cs}_{4} \mathrm{O}, \mathrm{Cs}_{11} \mathrm{O}_{3}$ und $\mathrm{Rb}_{9} \mathrm{O}_{2}$ has been measured with linearly polarised light in the range from 0.4 to $5 \mathrm{eV}$. The plasma frequency is derived as a function of the oxygen content by means of Kramers-Kronig analysis of the data. A Drude-Lorentz fit allows the assignment of additional structures in the spectra. These structures are discussed in terms of surface plasma oscillations, inter-band transitions from the $\mathrm{O} 2 \mathrm{p}$ band and "void modes".

\section{Einleitung}

Von den schweren Alkalimetallen Rubidium und Cäsium sind eine Reihe metallreicher Oxide (Suboxide) unterschiedlicher Zusammensetzung bekannt [1]. Mit Hilfe der Röntgen-Strukturanałyse, Messungen der Photoemission [2] und des elektrischen Widerstandes [3], sowie durch Modellrechnungen zur Stabilität geladener Cluster [4] konnte ein konsistentes Modell der chemischen Bindung in den Alkalimetallsuboxiden gewonnen werden. Danach handelt es sich um Metalle, in denen charakteristische Baugruppen mit den Zusammensetzungen $\mathrm{Cs}_{11} \mathrm{O}_{3}$ bzw. $\mathrm{Rb}_{9} \mathrm{O}_{2}$ vorliegen. Diese treten in den Verbindungen $\mathrm{Cs}_{11} \mathrm{O}_{3}$ und $\mathrm{Rb}_{9} \mathrm{O}_{2}$ ausschließlich auf (,komplexe Metalle"). In metallreicheren Verbindungen wie $\mathrm{Cs}_{7} \mathrm{O}\left(=\left(\mathrm{Cs}_{11} \mathrm{O}_{3}\right) \mathrm{Cs}_{10}\right), \mathrm{Rb}_{6} \mathrm{O}\left(=\left(\mathrm{Rb}_{9} \mathrm{O}_{2}\right) \mathrm{Rb}_{3}\right)$ oder $\left(\mathrm{Cs}_{11} \mathrm{O}_{3}\right) \mathrm{Rb}_{7}$ liegen diese Baugruppen neben stöchiometrischen Anteilen des jeweiligen Alkalimetalls vor. Die interatomaren Abstände legen eine Beschreibung der Baugruppen als Ionencluster im Sinne der Formulierung $\left(\mathrm{Cs}_{11} \mathrm{O}_{3}\right)^{5+} 5 \mathrm{e}^{-}$bzw. $\left(\mathrm{Rb}_{9} \mathrm{O}_{2}\right)^{5+} 5 \mathrm{e}^{-}$nahe [1]. UPS-Untersuchungen an Alkalimetallsuboxiden unterstützen diese Vorstellung [2]. Energieberechnungen für die Cluster auf der Basis eines einfachen elektrostatischen Modells zeigen darüber hinaus [4], daß Ionencluster mit den Ladungen $5+$ nicht stabil sind, sondern erst durch die zusätzlichen Elektronen stabilisiert werden. Diese bewirken eine partielle Abschirmung der positiven Ladungen und führen zu einer effektiv niedrigeren Cluster-Ladung.

Reprint requests to Herrn Prof. A Simon, Max-PlanckInstitut für Festkörperforschung, Heisenbergstr. 1, D-7000 Stuttgart 80.
Das Innere der $\mathrm{Cs}_{11} \mathrm{O}_{3}$ - bzw. $\mathrm{Rb}_{9} \mathrm{O}_{2}$-Cluster ist durch die Anhäufung von $\mathrm{O}^{2-}$-Ionen repulsiv für die Leitungselektronen. Betrachtet man die Clusterzentren vereinfacht als für Leitungselektronen verbotene Bereiche, so lassen sich die Alkalimetallsuboxide modellhaft als Metalle mit einer regelmäßigen Verteilung von Löchern ("void metals") beschreiben, deren Ausdehnung für den Fall des $\mathrm{Cs}_{11} \mathrm{O}_{3}$-Clusters etwa $8 \AA$ beträgt. Diese Besonderheit in den Kristallstrukturen läßt Besonderheiten in der elektronischen Struktur erwarten, die z. B. darin bestehen, daß bestimmte Wellenvektoren innerhalb der Fermi-Kugel fehlen. Nach Burt und Heine [5] ist die erniedrigte Austrittsarbeit der Suboxide gegenüber den reinen Alkalimetallen auf die Anwesenheit periodisch angeordneter isolierter Bereiche zurückzuführen.

Die optischen Eigenschaften eines Metalls, das nicht-wechselwirkende isolierende Bereiche (Löcher) enthält, können nach einem Modell von Genzel und Martin [6] berechnet werden. Als wesentliches Merkmal tritt eine Zusatzabsorption bei der Plasmafrequenz des ungestörten Metalls auf. Die vorliegende Untersuchung der Reflektivität an Suboxiden diente einerseits der Suche nach derartigen spezifischen Strukturen in der Nähe der Plasmakante und sollte frühere, vorläufige Messungen [7] sichern und erweitern, die zur Erklärung der charakteristischen Farben der Suboxide und zur Überprüfung des Bindungsmodells durchgeführt worden waren.

\section{Experimentelle Angaben}

\subsection{Probenherstellung, Probengefäße}

Die Herstellung der Ausgangsschmelzen und Methoden zur Kristallzüchtung wurden in früheren 
Arbeiten ausführlich beschrieben [8-13]. Die Kristallzüchtungsverfahren mußten aufgrund der wesentlich größeren Probenmengen zum Teil modifiziert werden. Diese abgeänderten Methoden führten auch zur reproduzierbaren Darstellung eines bisher vermuteten [8], jedoch röntgenographisch nicht faßbaren $\mathrm{Rb}$-Suboxids, das aufgrund der Farbe metallreicher als $\mathrm{Rb}_{6} \mathrm{O}$ sein sollte, bisher aber nicht genauer charakterisiert werden konnte.

Die große Reaktivität der Suboxide erfordert eine ständige Aufbewahrung der Proben unter hochreinem Schutzgas oder unter Vakuum. Es wäre daher naheliegend, den ganzen Meßaufbau unter Hochvakuum-Bedingungen $\mathrm{zu}$ erstellen, wodurch Probleme mit Fenstern vermieden und ein erweiterter Meßbereich ins Vakuum-UV erreicht würden. Andererseits entstehen dadurch ein größerer experimenteller Aufwand und erhebliche Schwierigkeiten bei Kristallzüchtung und -justierung. Es wurde deshalb die Alternative spezieller Probengefäße ge- wählt, die schematisch in Abb. 1 dargestellt sind. Die Suprasil-Gefäße wurden über den Einfüllstutzen mit den Ausgangsschmelzen beschickt; zur Kristallzüchtung wurden nach den Phasendiagrammen geeignete Probenzusammensetzungen und Temperaturen gewählt [8]. Die Probentemperierung erfolgte mit einem Ultrakryostaten TUK 30 (Fa. Lauda; $\pm 0,02{ }^{\circ} \mathrm{C}$ ) mit angeschlossenem Programmgeber. Die Kristalle hatten in der Regel Oberflächen von $1-8 \mathrm{~mm}^{2}$, die durch Bewegung des Probengefäßes senkrecht zum einfallenden Strahl justiert wurden. Im einzelnen galten die folgenden experimentellen Bedingungen.

Rb [8]: Gut spiegelnde Oberflächen wurden durch langsames Abkühlen $\left(1^{\circ} \mathrm{C} / \mathrm{h}\right)$ einer Schmelze erhalten. Die Messungen erfolgten bei $39^{\circ} \mathrm{C}$, d.h. $0,4^{\circ} \mathrm{C}$ unterhalb des Schmelzpunktes, da die Oberfläche bei tiefen Temperaturen durch Kontraktion des Metalles zunehmend rauher und konkav gekrümmt wird. a)

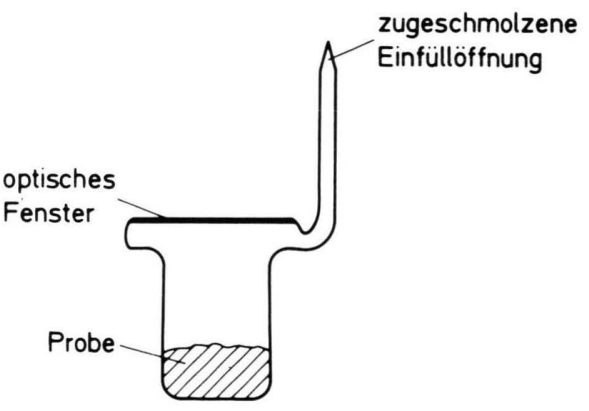

b)

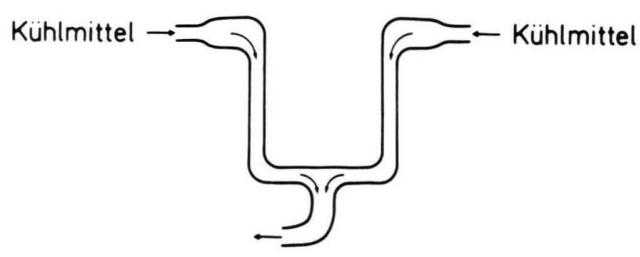

c)
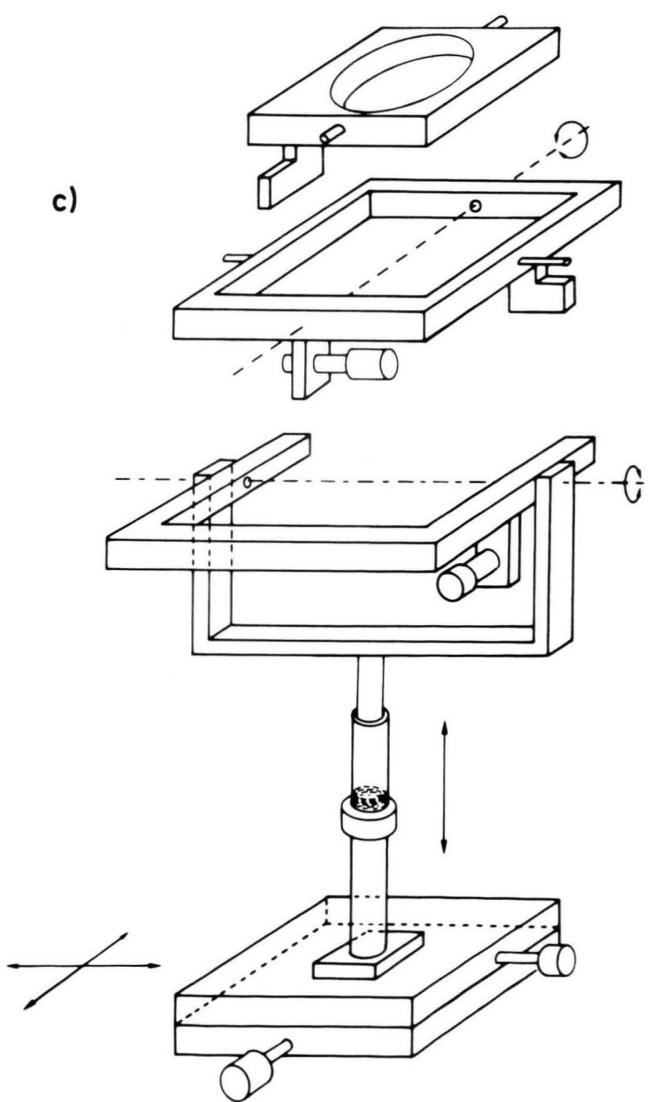

Abb. 1. a) Probengefäße für die Messung der Reflektivitäten von Alkalimetallsuboxiden, b) Temperiereinsatz für Probengefäße, c) Vorrichtung zur Orientierung der Probenoberfläche. 
$\mathbf{R b}_{\mathbf{9}} \mathbf{O}_{\mathbf{2}}$ [3]: Durch langsames $\left(1^{\circ} \mathrm{C} / \mathrm{h}\right)$ Abkühlen einer Schmelze $\mathrm{RbO}_{0,22}$ wurden unterhalb $40,2{ }^{\circ} \mathrm{C}$ große kupferfarbene, auf der metallreicheren Schmelze schwimmende Kristalle erhalten. Die für die Reflexionsmessung benutzte Kristallfläche hatte eine Größe von ca. $1 \mathrm{~cm}^{2}$.

$\mathbf{R b}_{\mathbf{6}} \mathbf{O}$ [11]: Goldfarbene Kristalle von $\mathrm{Rb}_{6} \mathrm{O}$ wurden durch Abschrecken der Probe in flüssigem $\mathrm{N}_{2}-$ die Erstarrung erfolgte innerhalb von etwa $10 \mathrm{Se}$ kunden - und anschließendes Tempern bei $-8^{\circ} \mathrm{C}$ erhalten. Es entstanden immer größere Anteile an $\mathrm{Rb}_{9} \mathrm{O}_{2}$, die beim Tempern auf Kosten der $\mathrm{Rb}_{6} \mathrm{O}$ Kristalle wuchsen. Die ca. $1 \mathrm{~mm}^{2}$ großen Oberflächen der erhaltenen Kristalle waren für Messungen ungeeignet; ihr Reflexionsvermögen war nur gering.

Cs [8]: Eine spiegelnde Fläche wurde wie bei $\mathrm{Rb}$ erhalten und bei $28^{\circ} \mathrm{C}\left(0,5^{\circ} \mathrm{C}\right.$ unterhalb des Schmelzpunktes) vermessen.

$\mathrm{Cs}_{7} \mathrm{O}$ [9]: Die in bronzefarbenen Nadeln mit ausgeprägter Unterkühlung kristallisierende Verbindung konnte nur mit sehr rauhen Kristalloberflächen erhalten werden. Bei einer Abkühlungsgeschwindigkeit von $1{ }^{\circ} \mathrm{C} / \mathrm{h}$ setzte die Kristallisation bei $-10^{\circ} \mathrm{C}$ (statt $4,3^{\circ} \mathrm{C}$ ) ein. Einzelne Kristalle hatten eine Länge von $6-8 \mathrm{~mm}$.

$\mathrm{Cs}_{\mathbf{4}} \mathrm{O}$ [10]: Durch Eintauchen des Probengefäßes mit einer Schmelze von $\mathrm{CsO}_{0,23}$ in $\mathrm{CO}_{2}$ /Ethanol entstand mikrokristallines $\mathrm{Cs}_{4} \mathrm{O}$, das in rotviolett erstarrte Restschmelze eingebettet war. Tagelanges pendelndes Tempern zwischen 8 und $10^{\circ} \mathrm{C}$ führte zur Vergrößerung der $\mathrm{Cs}_{4} \mathrm{O}$-Kristalle, die jedoch häufig in der Schmelze versanken. Die Messungen wurden mit der etwa $4 \mathrm{~mm}^{2}$ großen Fläche eines auf der Oberfläche der Schmelze schwimmenden Kristalls durchgeführt.

$\mathrm{Cs}_{11} \mathrm{O}_{3}$ [12]: Durch Abkühlung einer Schmelze $\mathrm{CsO}_{0,25}$ mit $1{ }^{\circ} \mathrm{C} / \mathrm{h}$ bildeten sich auf der Oberfläche schwimmende lattenförmige $\mathrm{Cs}_{11} \mathrm{O}_{3}$-Kristalle mit blauviolett spiegelnden Flächen aus. Merkwürdigerweise waren einige Kristalle mit goldfarbenen Flekken bedeckt. Die Messungen wurden mit einer Kristallfläche von ca. $5 \mathrm{~mm}^{2}$ vorgenommen.

“ $\mathrm{Cs}_{3} \mathrm{O}$ " [8]: Das sauerstoffreichste Cäsiumsuboxid besitzt ein etwa vom $\mathrm{CsO}_{0,31}$ bis $\mathrm{CsO}_{0,37}$ reichendes Homogenitätsgebiet und bildet fächerförmige Kristalle mit blaugrünem Metallglanz. Gut reflektierende Kristallflächen konnten nicht erhalten werden. Für die Messungen wurde eine Probe der Zusammensetzung $\mathrm{CsO}_{0,333}$ geschmolzen (ca. $170^{\circ} \mathrm{C}$ ) und durch Erstarren der Schmelze auf dem Fenster der Küvette ein (violettfarbener) Spiegel erzeugt.

\subsection{Reflektometer}

Der in Abb. 2 skizzierte optische Aufbau des verwendeten Reflektometers befindet sich auf einer schwingungsdämpfend gelagerten Granitplatte. Die Wendel der Lichtquelle $L$ wird über die beiden Hohlspiegel $\mathrm{H} 1$ und $\mathrm{H} 2$ auf den Eingangsspalt des Monochromators (HRS 2, JOBIN-YVON; holographische Gitter) abgebildet. Hinter dem Ausgangsspalt blenden entsprechende Kantenfilter $F$ das Licht höherer Ordnung aus. Über eine Zylinderlinse ZL gelangt das nun parallele, monochromatische Licht zum Hohlspiegel H3. Am Strahlteiler ST wird das Licht in den Proben- und den Referenzstrahl aufgespalten. Nach der Reflexion an der Probenoberfläche P bzw. am Referenzspiegel R gelangt das Licht zum Hohlspiegel H4, der das Bild des Ausgangsspaltes auf die sensitive Fläche des Detektors PM abbildet. Durch den Chopper $\mathrm{CH}$ werden Proben- und Referenzstrahl abwechselnd ausgeblendet, so daß der Detektor ein getaktetes Signal registriert,

a)

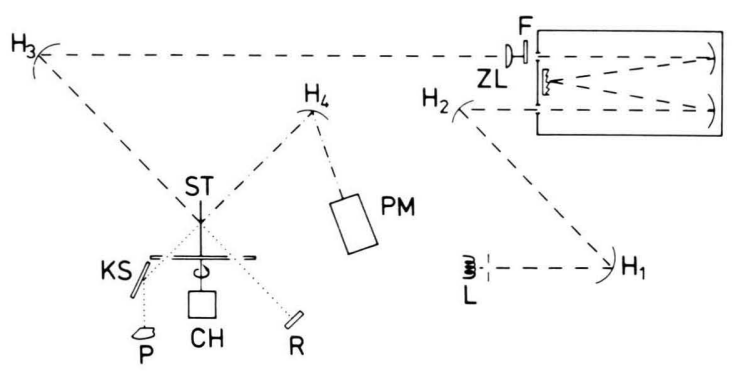

b)

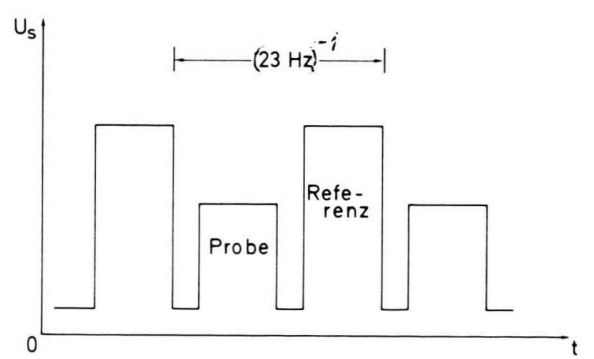

Abb. 2. a) Blockbild des Reflektometers, b) Meßsignale. 
das in einer Halbperiode das Probensignal, in der anderen das Referenzsignal enthält.

Durch diese Anordnung können Proben- und Referenzssignal annähernd gleichzeitig gemessen werden, so daß Intensitätsschwankungen der Lampe das gemessene Reflexionsvermögen kaum noch verfälschen können. Außerdem genügt zur Signalaufnahme ein Detektor. Dadurch entfällt auch der Einfluß unterschiedlicher Nachweischarakteristika verschiedener Detektoren. Bis auf den zusätzlichen Kippspiegel KS, ohne den keine gekühlten oder flüssigen Proben gemessen werden können, sind die optischen Wege in Referenz- und Probenstrahlengang identisch. Deshalb genügt es, das gemessene Probenreflexionsvermögen mit einer Eichmessung für den Aluminiumkippspiegel zu korrigieren. Alle anderen optischen Bauelemente kompensieren sich in ihrer Wirkung auf das gemessene Reflexionsvermögen.

$\mathrm{Zu}$ Beginn der Messungen wird das Probengefäß in ein doppelwandiges Glasgefäß eingesetzt. Dieses Glasgefäß ist über Kühlschläuche mit dem Ultrakryostaten verbunden (Abbildung 1). Um ein Beschlagen des optischen Fensters im Probengefäß zu verhindern, wird es während der Messung durch Anblasen mit warmer Luft auf Zimmertemperatur gehalten.

Proben- und Kühlgefäß hängen in einer kombinierten Kipp-Verschiebe-Mechanik, die eine exakte Justierung der Probenoberfläche im Strahlengang erlaubt (Abbildung 1 c).

Die Größe des abzudeckenden Spektralbereichs erfordert eine Unterteilung in mehrere Teilbereiche, die durch die spektralen Eigenschaften der verwendeten Lampen, Gitter, Detektoren und Filter festgelegt werden (Tabelle 1).

Das gemessene Reflexionsvermögen entspricht dem Produkt aus Probenreflektivität und Reflektivität des Aluminiumkippspiegels

$$
R_{\text {Probe }}=R_{\text {Mess }} / R_{\mathrm{Al}} \text {. }
$$

Das Reflexionsvermögen des Aluminiumkippspiegels wurde durch mehrere Eichmessungen bestimmt. Dazu wurden als Probe, als Referenz- und als Kippspiegel drei nach demselben Verfahren aufgedampfte Aluminiumspiegel benutzt. Die bei Testmessungen an Al-Spiegeln erhaltenen Reflexionsdaten stimmten gut mit Literaturwerten [14] überein (Abbildung 3).

Die Kramers-Kronig-Analyse [15] sowie die Anpassungsrechnungen wurden mit dem Honeywell

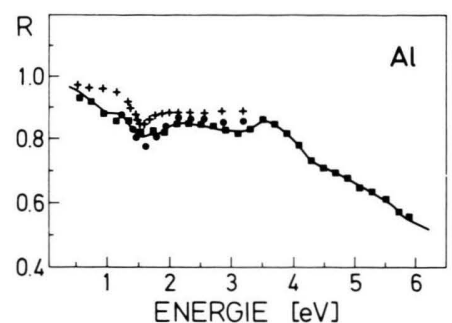

Abb. 3. Reflexionsspektren von Aluminium, +: Bennett, Silver, Ashley; $\bullet$ : Köhler [14]. Die durch die quadratischen Meßpunkte gezogene Linie gibt das in der vorliegenden Arbeit gemessene Spektrum wieder.

Bull-Rechner 66/80 Dual der Max-Planck-Institute Stuttgart durchgeführt. Verwendet wurde das Minimalisierungsprogramm MINUIT aus der CERN computer program library (F. James and M. Roos).

\section{Ergebnisse und Diskussion}

Die Reflexionsspektren der Metalle $\mathrm{Rb}$ und $\mathrm{Cs}$ sowie ihrer Suboxide sind in Abb. 4 zusammengestellt. Bei Messungen mit polarisiertem Licht ergeben sich teilweise erhebliche Unterschiede in den Spektren. Die Bezeichnungen $\|$ und $\perp$ entsprechen senkrecht zueinander stehenden Polarisationsebenen; gewöhnlich bedeutet $\|$ Parallelität zur größten Ausdehnung des Kristalls. Bei nadelförmig kristallisierenden Substanzen wie $\mathrm{Cs}_{7} \mathrm{O}$ und $\mathrm{Cs}_{11} \mathrm{O}_{3}$ kann die Polarisationsrichtung kristallographischen Achsen zugeordnet werden: $\mathrm{Bei} \mathrm{Cs}_{7} \mathrm{O}$ bedeutet parallel zur $c$-Achse, bei $\mathrm{Cs}_{11} \mathrm{O}_{3}$ parallel zur $b$-Achse. Es liegt nahe, die Polarisationsabhängigkeit der Spektren mit der Anisotropie der jeweiligen Strukturen in Verbindung zu bringen. Dafür spricht einerseits das weitgehende Fehlen dieses Effektes bei den reinen

Tab. 1. Angaben zu den spektralen Bereichen.

\begin{tabular}{lllll}
\hline Bereich $(\mathrm{nm})$ & $\begin{array}{l}\text { Detek- } \\
\text { tor }\end{array}$ & Lampe & $\begin{array}{l}\text { Gitter- } \\
\text { strichzahl }\end{array}$ & KF \\
\hline $200-350$ & PM & Deuterium & 1200 & - \\
$330-590$ & PM & Wolfram & 1200 & $360 \mathrm{~nm}$ \\
$580-750$ & PM & Wolfram & 1200 & $590 \mathrm{~nm}$ \\
$700-920$ & $\mathrm{PM}$ & Wolfram & 600 & $590 \mathrm{~nm}$ \\
$880-1150$ & PbS & Wolfram & 600 & $590 \mathrm{~nm}$ \\
$1070-1780$ & PbS & Wolfram & 600 & $\mathrm{Si}$ \\
$1650-3500$ & PbS & Wolfram & 300 & $\mathrm{Ge}$ \\
\hline
\end{tabular}

PM: Photomultiplier, PbS: Bleisulfid-Zelle, Si: Siliziumscheibe, Ge: Germaniumscheibe, KF: Kantenfilter. 

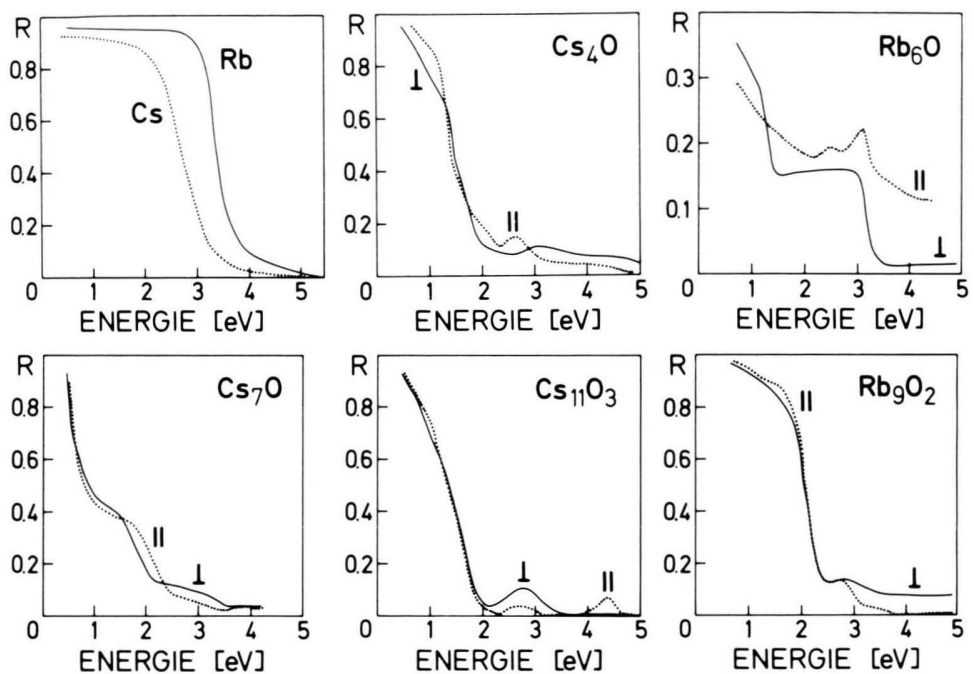

ENERGIE [eV]
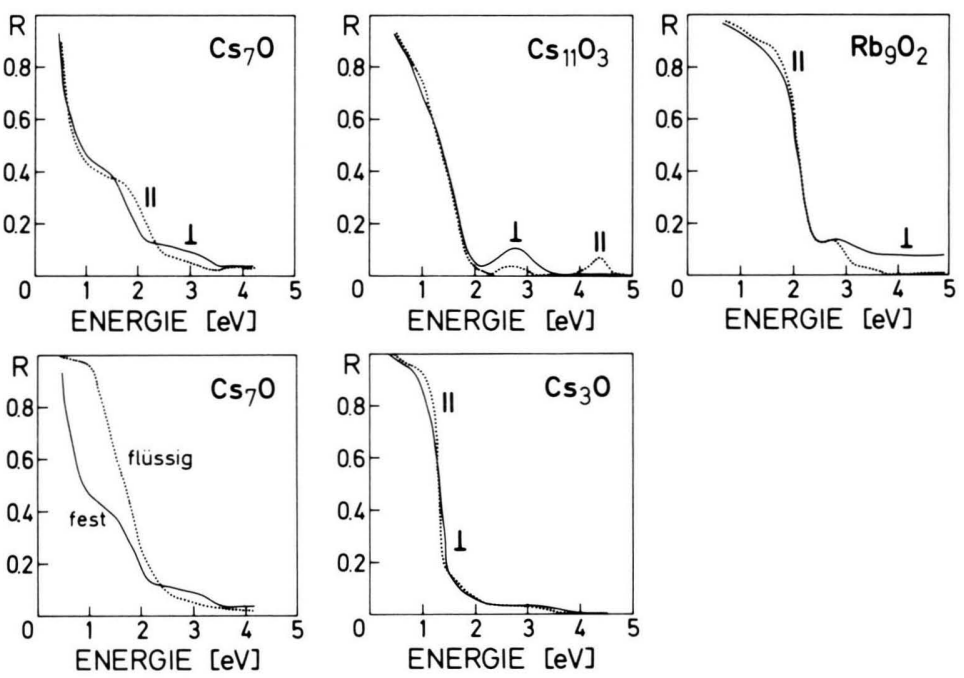

ENERGIE [eV]

Abb. 4. Gemessene Reflexionsspektren an Einkristallen von $\mathrm{Rb}$, Cs und deren Suboxiden. Die Bezeichnungen $\|$ bzw. $\perp$ stehen für eine Orientierung des Lichtvektors parallel bzw. senkrecht zur größten Ausdehnung der Kristalle.

Metallen $\mathrm{Rb}$ und $\mathrm{Cs}$. Auch der gravierende Unterschied der Reflexionsspektren von festem und flüssigem $\mathrm{Cs}_{7} \mathrm{O}$ spricht für diese Deutung. Andererseits waren die Oberflächen der $\mathrm{Cs}_{7} \mathrm{O}$-Kristalle stets gestört, und die Polarisationsabhängigkeit könnte auch mit der Streifung der vermessenen Kristallflächen zusammenängen. Für diese triviale Deutung

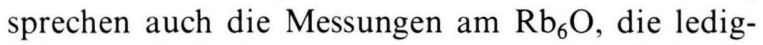
lich ein Reflexionsvermögen von etwa $30 \%$ der erwarteten metallischen Werte zeigen und besonders ausgeprägte Polarisationsabhängigkeit erkennen lassen.

Die Reflektivität der Alkalimetallsuboxide entspricht einem für Metalle typischen Verhalten. Bei niedrigen Energien liegt hohe Reflektivität vor, die in Form einer Plasmakante in einem Bereich geringer Reflektivität mit schwach ausgeprägten Strukturen übergeht. Während diese Strukturen auf Anregungen gebundener Elektronen zurückzuführen sind, wird der niederenergetische Bereich sowie die Plasmakante im wesentlichen durch freie Ladungsträger bestimmt, deren Konzentration sich mit stei- gendem Sauerstoffgehalt verringert. Ohne genauere Analyse ist die Verschiebung der Plasmakante zu kleineren Energien von $\mathrm{Rb}$ (silberfarben) $\mathrm{zu}^{\mathrm{R} b_{9} \mathrm{O}_{2}}$ (kupferfarben) zu erkennen und damit der Farbwechsel mit der abnehmenden Ladungsträgerkonzentration zu erklären. Reines Cs, d.h. Metall mit weniger als 2 ppm Sauerstoff als Verunreinigung, ist nach früheren Untersuchungen bereits goldfarben. Auch bei den Cs-Suboxiden erklärt die Verschiebung der Plasmakante zu kleineren Energien die charakteristischen Farben der Verbindungen. Der einfache Zusammenhang zwischen Ladungsträgerkonzentration und Lage der Plasmakante wird allerdings durch die zusätzlichen Strukturen in der Nähe der Plasmakanten der Suboxide kompliziert. Die Kramers-Kronig (KK)-Analyse der Spektren macht dies deutlicher. In den Energieverlustfunktionen $\left(\varepsilon_{2} /\left(\varepsilon_{1}^{2}+\varepsilon_{2}^{2}\right) ; \varepsilon_{1,2}\right.$ Real- bzw. Imaginärteil der Dielektrizitätsfunktion) existieren nur bei den reinen Metallen $\mathrm{Rb}$ und $\mathrm{Cs}$ einfache scharfe Maxima, die Plasmaresonanzen bei $E_{\mathrm{p}}=3,44$ und $E_{\mathrm{p}}=2,88$ $\mathrm{eV}$ zuzuordnen sind (Abbildung 5). Diese Werte 
stimmen gut mit Literaturangaben überein, die im Bereich 2,8-3 eV für $\mathrm{Cs}$ und 3,3 bis $3,4 \mathrm{eV}$ für $\mathrm{Rb}$ liegen [16]. Geringe Abweichungen vom freienElektronen-Verhalten deuten sich jedoch auch hier bereits in der Asymmetrie der Strukturen an.

Abbildung 6 zeigt den erheblich komplizierteren Verlauf der Energieverlustfunktionen bei den Alkalimetall-Suboxiden. Auf die Wiedergabe des Ergebnisses für $\mathrm{Rb}_{6} \mathrm{O}$ wurde wegen der erwähnten Meßprobleme verzichtet. Doch auch in anderen Fällen, beispielsweise bei $\mathrm{Cs}_{4} \mathrm{O}$, stößt die direkte Bestimmung der Plasmaenergie aus den gemessenen Spektren auf Probleme.

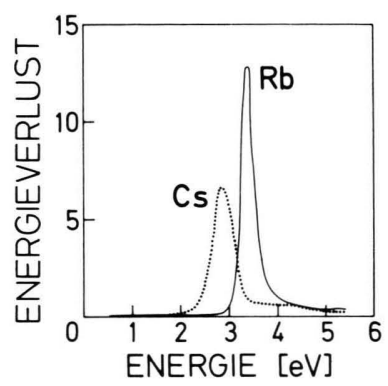

Abb. 5. Energieverlustfunktionen von $\mathrm{Rb}$ und $\mathrm{Cs}$, berechnet aus den gemessenen Reflexionsdaten.
Im folgenden soll der Versuch gemacht werden, zu einem weitergehenden Verständnis der Spektren durch Anpassung der Reflexionsdaten an geeignete Modelle zu kommen. Als solche bieten sich das allgemein für Metalle geltende Drude-Lorentz-Modell [17] an sowie das eingangs erwähnte "LöcherModell", mit dem möglicherweise die strukturellen Eigenheiten der Suboxide beschreibbar sind.

\subsection{Drude-Lorentz-Modell}

Das Drude-Lorentz (DL)-Modell beschreibt das Reflexionsvermögen von Metallen nur für den infraroten Spektralbereich gut. Im Bereich sichtbaren Lichts bzw. im UV-Bereich, in denen Beiträge gebundener Elektronen hinzukommen bzw. dominieren, können die entsprechenden Resonanzen quantitativ nur mit der Quantenmechanik behandelt werden.

Im DL-Modell werden die Beiträge der Elektronen zur Dielektrizitätsfunktion $\varepsilon(\omega)$ durch einen Drude-Ansatz

$$
\varepsilon_{\mathrm{f}}(\omega)=1-\frac{\omega_{\mathrm{p}}^{2}}{\omega^{2}-\mathrm{i} \omega \omega_{\mathrm{D}}}
$$
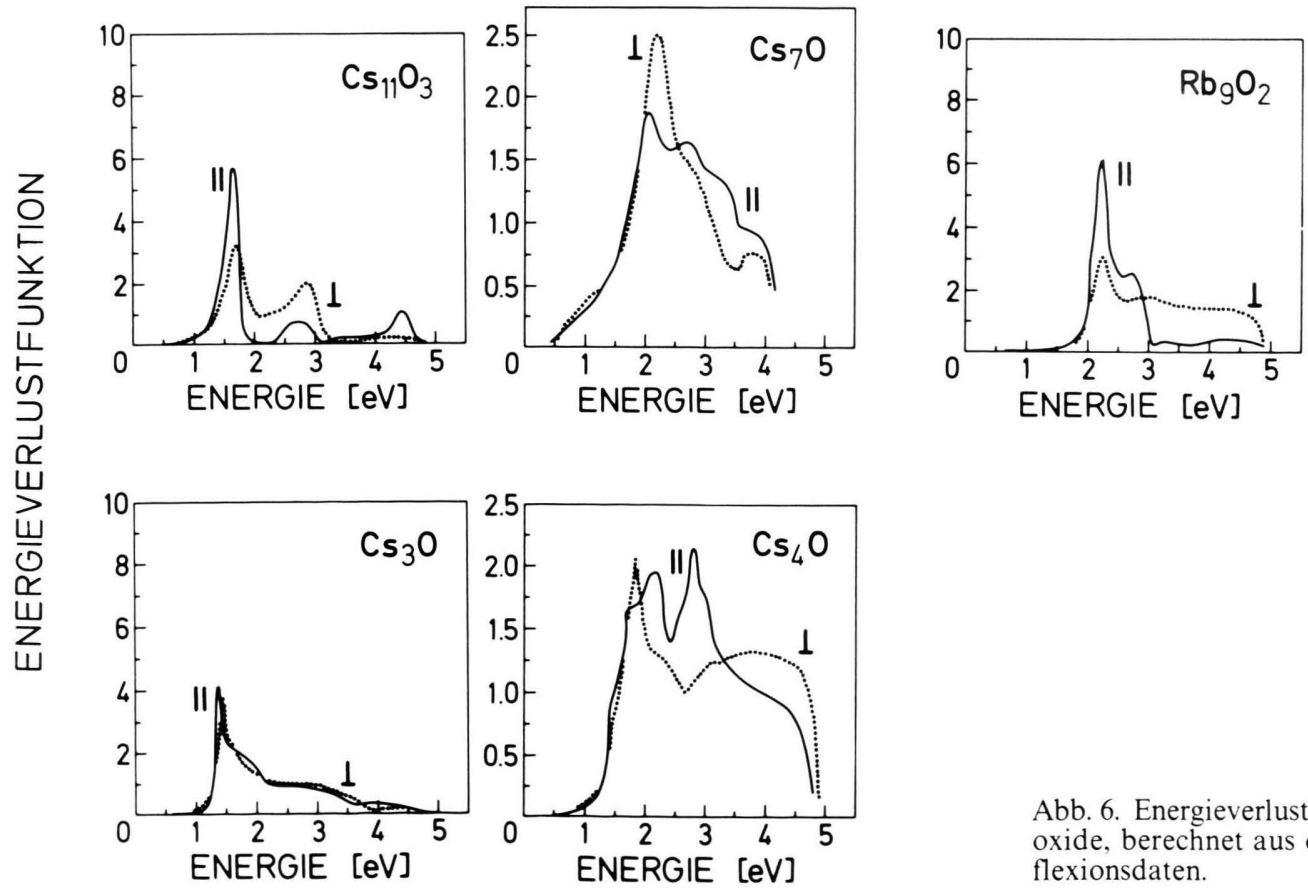

Abb. 6. Energieverlustfunktionen der Suboxide, berechnet aus den gemessenen Reflexionsdaten. 
für die freien Elektronen und eine Summe über gedämpfte Oszillatoren

$$
\varepsilon_{\mathrm{g}}(\omega)=\sum_{e} \frac{f_{\mathrm{e}} \omega_{\mathrm{e}}^{2}}{\left(\omega_{\mathrm{e}}^{2}-\omega^{2}\right)-i \omega \gamma_{\mathrm{e}}}
$$

für gebundene Elektronen (Interbandübergänge) beschrieben. Dabei sind

$$
\omega_{\mathrm{p}}^{2}=\frac{4 \pi N_{\mathrm{f}} e^{2}}{m_{\mathrm{eff}} \varepsilon_{\infty}}
$$

das Quadrat der Plasmafrequenz $\omega_{\mathrm{p}}, N_{\mathrm{f}}$ die Dichte der freien Ladungsträger, $m_{\text {eff }}$ deren effektive Masse, $\varepsilon_{\infty}=\varepsilon(\omega \rightarrow \infty), \omega_{\mathrm{e}}$ die Eigenfrequenzen der Oszillatoren, $f_{\mathrm{e}}$ deren Oszillatorstärken und $\omega_{\mathrm{D}}$ bzw. $\gamma_{\mathrm{e}}$ Dämpfungskonstanten.

Eine DL-Anpassung ist nur bei einer möglichst geringen Anzahl von Oszillatoren aussagekräftig. Die Reflexionsspektren der Suboxide lassen sich mit 4 Oszillatoren im allgemeinen recht gut anpassen. In der Abb. 7 sind die gemessenen Reflexionsdaten zusammen mit DL-angepaßten Spektren dargestellt. Zugunsten einer besseren Übersicht sind die gemessenen Spektren in Abb. 7 durch repräsentative Punkte dargestellt. Die tatsächliche Energieauflösung betrug weniger als $0,01 \mathrm{eV}$. Die Anpassungsparameter sind in Tab. 2 wiedergegeben. Die Anpassung der Spektren für verschiedene Suboxide erfolgte zunächst unabhängig voneinander. In einem zweiten Schritt wurden die jeweiligen Energiewerte fixiert, sofern sie sich für verschiedene Verbindungen um nicht mehr als $0,1 \mathrm{eV}$ unterschieden. Diesem Vorgehen entspricht die Annahme, daß ähnliche Absorptionsmechanismen für die Rb- bzw. CsSuboxide zu vermuten sind.

Abbildung 8 zeigt die mit der DL-Anpassung erhaltenen Energieverlustfunktionen. Absorptionsstellen sollten sich als Maxima in der Energieverlustfunktion zeigen. Sowohl die energetische Lage wie die Intensität sind in befriedigender Übereinstimmung mit den Maxima, die sich durch KK-Analyse der gemessenen Spektren (Abb. 6) ergeben. Die jeweils bei kleinsten Energiewerten in den Energieverlustfunktionen auftretenden Maxima entsprechen den Plasmaresonanzen. $\mathrm{Rb}_{6} \mathrm{O}$ und $\mathrm{Cs}_{7} \mathrm{O}$ weisen die größten Polarisationsabhängigkeiten der Plasmaenergien auf. Die nur geringe Reflektivität (insbesondere des $\mathrm{Rb}_{6} \mathrm{O}$ ) erlaubt allerdings keine Diskussion dieses Effektes im Zusammenhang mit den Kristallstrukturen. Aus den Plasmafrequenzen entsprechend den Maxima niedrigster Energie der Energieverlustfunktionen, die mit Hilfe einer KK-Analyse aus den gemessenen Reflexionsspektren erhalten wurden, kann über die Beziehung (3) die Dichte der freien Elektronen berechnet werden. Darin beschreibt $\varepsilon_{\infty}$ den Beitrag der gebundenen Elektronen und kann aus der DL-Anpassung zu maximal 1,2 abgeschätzt werden, so daß die Verwendung des Wertes 1 für $\varepsilon_{\infty}$ gerechtfertigt ist. Mit $m_{\text {eff }}=m_{0}$ ergeben sich die in

\begin{tabular}{|c|c|c|c|c|c|c|c|c|c|c|c|c|c|c|c|c|c|}
\hline $\begin{array}{l}\text { Sub- } \\
\text { stanz }\end{array}$ & $\begin{array}{l}E_{\mathrm{p}}=\hbar \omega_{\mathrm{p}} \\
{[\mathrm{eV}]}\end{array}$ & $\begin{array}{l}\omega_{\mathrm{D}} \\
{[\mathrm{eV}]}\end{array}$ & $\begin{array}{l}\mathrm{E} 01 \\
{[\mathrm{eV}]}\end{array}$ & $f_{1}$ & $\begin{array}{l}\gamma_{1} \\
{[\mathrm{eV}]}\end{array}$ & $\begin{array}{l}\mathrm{E} 02 \\
{[\mathrm{eV}]}\end{array}$ & $f_{2}$ & $\begin{array}{l}\gamma_{2} \\
{[\mathrm{eV}]}\end{array}$ & $\begin{array}{l}\mathrm{E} 03 \\
{[\mathrm{eV}]}\end{array}$ & $f_{3}$ & $\begin{array}{l}\gamma_{3} \\
{[\mathrm{eV}]}\end{array}$ & $\begin{array}{l}\mathrm{E} 04 \\
{[\mathrm{eV}]}\end{array}$ & $f_{4}$ & $\begin{array}{l}\gamma_{4} \\
{[\mathrm{eV}]}\end{array}$ & $\begin{array}{l}\mathrm{E} 05 \\
{[\mathrm{eV}]}\end{array}$ & $f_{5}$ & $\begin{array}{l}\gamma_{5} \\
{[\mathrm{eV}]}\end{array}$ \\
\hline \multicolumn{18}{|c|}{ Polarisation: $\perp$} \\
\hline Cs & 2,78 & 0,12 & - & - & - & - & - & - & 2,7 & 0,03 & 0,78 & - & - & - & - & - & - \\
\hline $\mathrm{Cs}_{7} \mathrm{O}$ & 2,14 & 0,63 & 1,3 & 0,18 & 0,5 & 2,5 & 0,03 & 0,9 & 2,8 & 0,04 & 0,5 & 4,0 & 0,05 & 0,8 & - & - & - \\
\hline $\mathrm{Cs}_{4} \mathrm{O}$ & 1,95 & 0,1 & 1,2 & 0,21 & 0,5 & 2,1 & 0,13 & 0,8 & 2,8 & 0,08 & 0,8 & 4,0 & 0,06 & 0,4 & - & - & - \\
\hline $\mathrm{Cs}_{11} \mathrm{O}_{3}$ & 1,73 & 0,1 & 1,1 & 0,33 & 0,5 & 2,5 & 0,14 & 0,4 & 2,8 & 0,06 & 0,5 & 4,25 & 0,03 & 0,9 & - & - & - \\
\hline $\mathrm{Cs}_{3} \mathrm{O}$ & 1,48 & 0,07 & 0,9 & 0,12 & 0,8 & 2,2 & 0,10 & 0,4 & 2,7 & 0,06 & 0,6 & 3,5 & 0,05 & 0,8 & 1,6 & 0,04 & 0,1 \\
\hline \multicolumn{18}{|c|}{ Polarisation: \|| } \\
\hline Cs & 2,78 & 0,12 & - & - & - & - & - & - & 2,7 & 0,03 & 0,78 & - & - & - & - & - & - \\
\hline $\mathrm{Cs}_{7} \mathrm{O}$ & 2,45 & 0,96 & 1,3 & 0,06 & 0,3 & 2,5 & 0,03 & 0,5 & 2,9 & 0,04 & 0,6 & 4,0 & 0,05 & 0,8 & - & - & - \\
\hline $\mathrm{Cs}_{4} \mathrm{O}$ & 1,95 & 0,71 & 1,2 & 0,21 & 0,5 & 2,0 & 0,17 & 0,4 & 2,5 & 0,10 & 0,5 & 4,0 & 0,06 & 0,4 & - & - & - \\
\hline $\mathrm{Cs}_{11} \mathrm{O}_{3}$ & 1,73 & 0,1 & 1,1 & 0,16 & 0,9 & 2,5 & 0,09 & 0,6 & 2,8 & 0,04 & 0,6 & 4,25 & 0,04 & 0,3 & - & - & - \\
\hline $\mathrm{Cs}_{3} \mathrm{O}$ & 1,41 & 0,05 & 0,9 & 0,12 & 0,6 & 2,2 & 0,10 & 0,4 & 2,7 & 0,06 & 0,6 & 3,5 & 0,03 & - & 1,6 & 0,04 & 0,1 \\
\hline \multicolumn{18}{|c|}{ Polarisation: $\perp$} \\
\hline $\mathrm{Rb}$ & 3,35 & 0,05 & - & - & - & - & - & - & - & - & - & 3,4 & 0,01 & 0,4 & - & - & - \\
\hline $\mathrm{Rb}_{9} \mathrm{O}_{2}$ & 2,41 & 0,06 & - & - & - & 1,1 & 0,17 & 0,9 & 2,6 & 0,15 & 0,5 & 3,4 & 0,09 & 0,6 & 4,0 & 0,06 & 0,8 \\
\hline \multicolumn{18}{|c|}{ Polarisation: $\|$} \\
\hline $\mathrm{Rb}$ & 3,35 & 0,05 & - & - & - & - & - & - & - & - & - & 3,4 & 0,01 & 0,4 & - & - & - \\
\hline $\mathrm{Rb}_{9} \mathrm{O}_{2}$ & 2,28 & 0,04 & - & - & - & 1,1 & 0,16 & 0,9 & 2,6 & 0,06 & 0,5 & 3,4 & 0,01 & 0,06 & 4,0 & 0,12 & 0,8 \\
\hline
\end{tabular}

Tab. 2. Angepaßte Parameter für die Alkalimetallsuboxide nach dem Drude-Lorentz-Modell. 

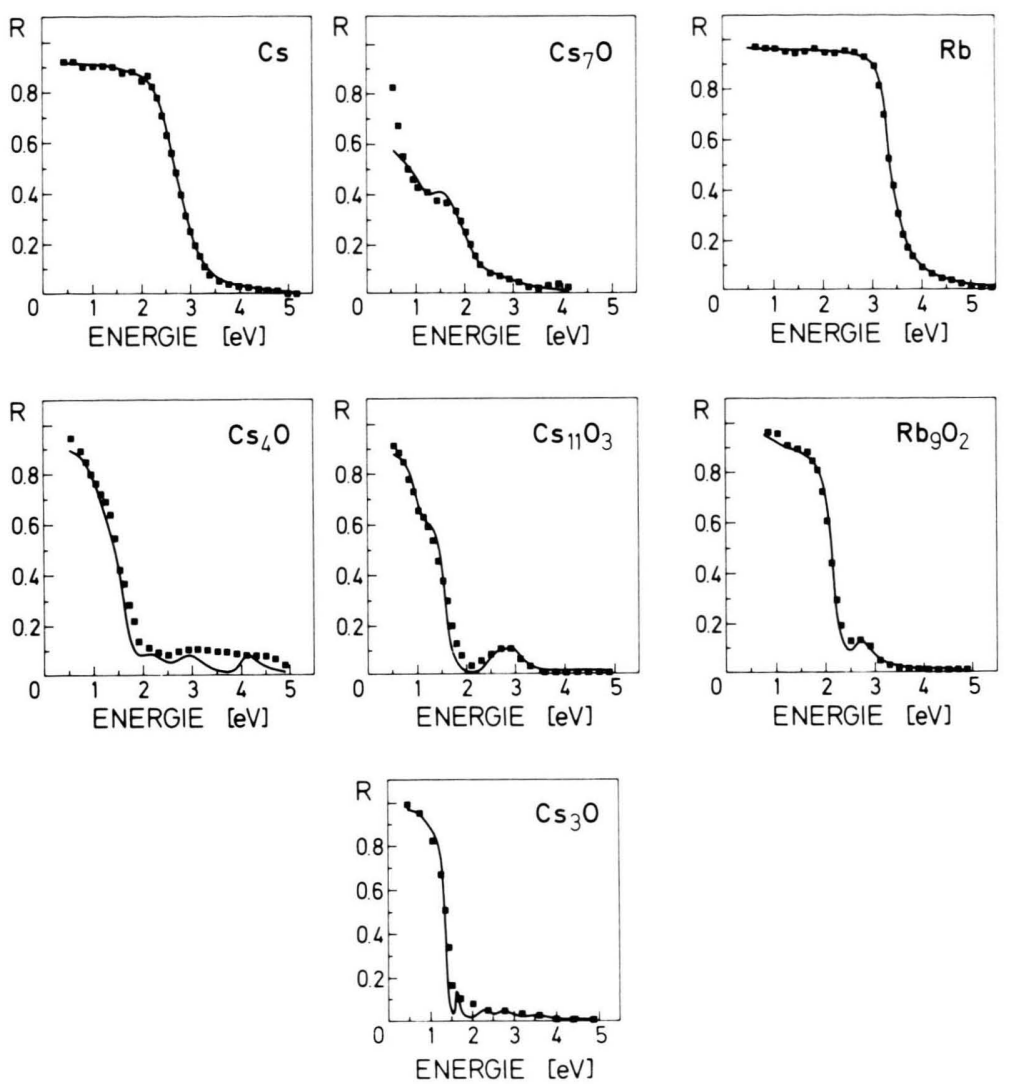

Abb. 7. Anpassung der gemessenen Reflexionsspektren (schwarze Punkte; Polarisation |) nach dem Drude-LorentzModell. Anpassungsparameter siehe Tabelle 2 .
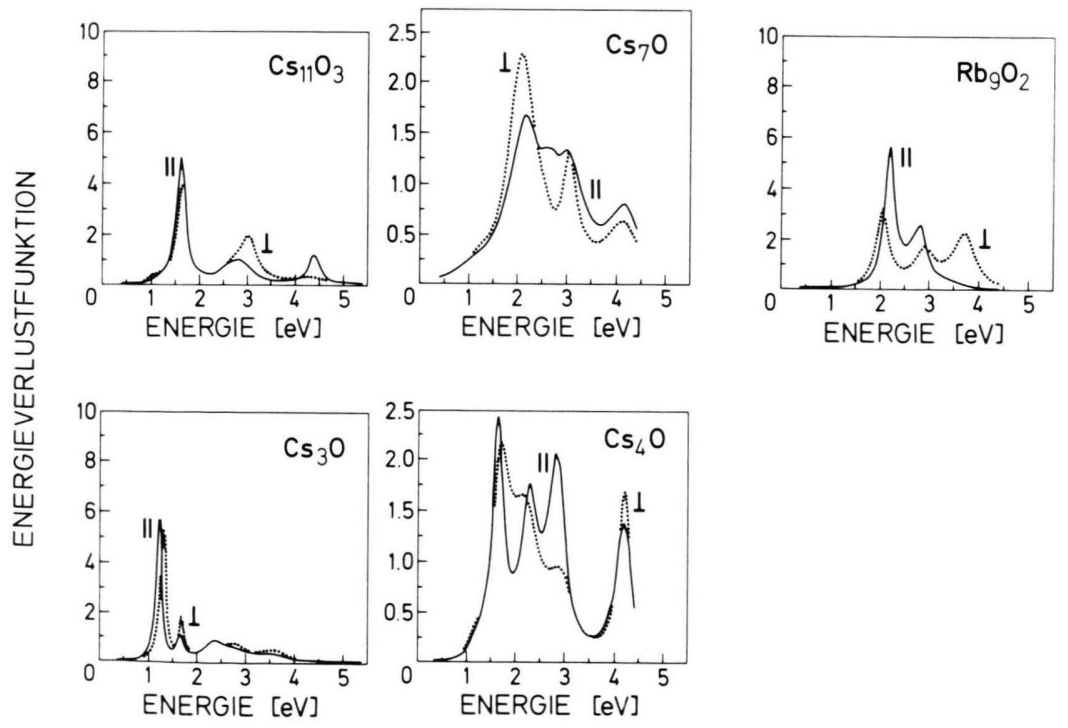

Abb. 8. Energieverlustfunktionen der Suboxide, berechnet aus den Drude-Lorentz-Anpassungen. 
Tab. 3. Plasmaenergien und Elektronendichten entsprechend den Maxima niedrigster Energie der Energieverlustfunktionen (berechnet mit Hilfe einer KK-Analyse aus den gemessenen Reflexionsspektren) im Vergleich mit Bindungsbetrachtungen und Stabilitätsüberlegungen. FE: Formeleinheit; e/FE: Elektronen pro Formeleinheit.

\begin{tabular}{lcccccc}
\hline Substanz & $\mathrm{Cs}$ & $\begin{array}{l}\mathrm{Cs}_{11} \mathrm{O}_{3} \mathrm{Cs}_{10} \\
\left(\hat{=} \mathrm{Cs}_{7} \mathrm{O}\right)\end{array}$ & $\begin{array}{l}\mathrm{Cs}_{11} \mathrm{O}_{3} \mathrm{Cs} \\
\left(\hat{=} \mathrm{Cs}_{4} \mathrm{O}\right)\end{array}$ & $\mathrm{Cs}_{11} \mathrm{O}_{3}$ & $\mathrm{Rb}$ & $\mathrm{Rb}_{9} \mathrm{O}_{2}$ \\
\hline$V_{\mathrm{FE}}\left[\AA^{3}\right]$ & 111 & 2095 & 1046 & 960 & 89 & 633 \\
$E_{\mathrm{p}}[\mathrm{eV}]$ & 2.88 & 2.28 & 1.76 & 1,61 & 3,42 & 2,19 \\
$N_{\mathrm{f}}\left[10^{21} \mathrm{~cm}^{-3}\right]$ & 6,01 & 3.14 & 2,25 & 1,88 & 8,48 & 3,48 \\
$\mathrm{e} / \mathrm{FE}$ & 0,67 & 7,2 & 2,4 & 1,8 & 0,76 & 2,3 \\
\hline
\end{tabular}

Tab. 3 zusammengefaßten Werte. Die aus den Plasmafrequenzen erhaltenen Werte für die Zahl der freien Elektronen pro Formeleinheit liegen im allgemeinen erheblich unter denen, die mit der Annahme von 5+ geladenen $\mathrm{Cs}_{11} \mathrm{O}_{3}-$ bzw. $\mathrm{Rb}_{9} \mathrm{O}_{2}$ Clustern berechnet werden. Die Abweichungen können durch Variation von $\varepsilon_{\infty}$ bzw. $m_{\text {eff }}$ in realistischen Grenzen nicht korrigiert werden. Vergleichende Messungen von Endriz und Spicer [18] zeigen, daß aus Reflexionsdaten ermittelte Plasmafrequenzen kleiner als die aus Photoemissionsmessungen bestimmten Werte sind. Dies gilt nicht für die vorliegenden Messungen an den reinen Metallen $\mathrm{Rb}$ und Cs, für die die Plasmaenergien aus Reflexionsmessungen und die Oberflächenplasmon-Energien sich wie $\sqrt{2}: 1$ verhalten. Dagegen sind die für die Suboxide aus den Reflexionsdaten ermittelten Plasmafrequenzen (bzw. Konzentrationen freier Ladungsträger) drastisch gegenüber den Erwartungswerten für ein reines Ionencluster-Modell erniedrigt. Möglicherweise ist dieser Befund mit den Ergebnissen von Rechnungen zur Stabilität der Ionencluster zu verstehen [4]. Danach sind Cluster mit der Ladung +5 nicht stabil; dies wird erst durch eine Abschirmung der Ladung bis auf etwa $+2,4$ für $\mathrm{Rb}_{9} \mathrm{O}_{2}$ (1,9 für $\mathrm{Cs}_{11} \mathrm{O}_{3}$ ) erreicht. Die zur Abschirmung benötigten zusätzlichen Elektronen sind möglicherweise weitgehend an die Cluster gebunden, so daß sie nicht mehr zur Plasmaschwingung der freien Elektronen beitragen. In jedem Fall stellt die Annahme einer homogenen Dichte der freien Elektronen eine erhebliche Vereinfachung dar.

Die DL-Anpassung der Reflexionsdaten der Alkalimetallsuboxide ergibt Oszillatoren, die anhand der Ergebnisse von Photoemissionsmessungen diskutiert werden können. Die Energiewerte für EO1 sind etwa um $\sqrt{2}$ kleiner als die zugehörigen Plasmafrequenzen und können demzufolge Oberflächenplasmonen an der Grenzfläche Suboxid-Vakuum zugeordnet werden.

Bei allen untersuchten Suboxiden liefert die DLAnpassung einen Oszillator EO3 bei etwa 2,7 eV.
Diese Energie entspricht der Anregung eines Elektrons aus einem O2p-Niveau zur Fermikante. Allerdings weist Cäsium selbst bei dieser Energie einen Oszillator vergleichbarer Stärke auf (Tabelle 2), so daß bei gleichem Energiewert ein Cs-Interbandübergang vorliegen muß. Das im nächsten Abschnitt diskutierte Löchermodell liefert ebenfalls eine Beitrag im gleichen Energiebereich. Eine eindeutige Zuordnung des EO3-Oszillators zu einem der drei beschriebenen Absorptionsmechanismen ist schon wegen der gegenläufigen Abhängigkeit der Oszillatorstärke vom Sauerstoffgehalt der Proben nicht möglich.

\subsection{Löchermodell}

Die Alkalimetallsuboxide stellen aufgrund ihrer strukturellen Gemeinsamkeiten Modellsysteme für "void metals" dar: Ionisch gebundene Cluster (Isolatorbereiche) sind in eine metallische Matrix eingefügt. Genzel und Martin [6] haben im Rahmen eines Kontinuumsmodells die Dielektrizitätsfunktion $\varepsilon_{\mathrm{av}}(\omega)$ eines solchen zusammengesetzten Mediums berechnet:

$$
\begin{aligned}
& \varepsilon_{\mathrm{av}}(\omega) \\
& \quad=\varepsilon_{\mathrm{m}}(\omega) \frac{\varepsilon v+(1-v) g \varepsilon+(1-v)(1-g) \varepsilon_{\mathrm{m}}(\omega)}{v \varepsilon_{\mathrm{m}}+(1-v) g \varepsilon+(1-v)(1-g) \varepsilon_{\mathrm{m}}(\omega)} .
\end{aligned}
$$

Dabei ist $\varepsilon_{\mathrm{m}}(\omega)$ die Dielektrizitätsfunktion des Metalls, $\varepsilon$ die Dielektrizitätsfunktion der Isolatorbereiche, $v$ der Volumenanteil der Isolatorbereiche und $g$ der Entelektrisierungsfaktor der Isolatorbereiche parallel zum äußeren Feld.

Dem Löchermodell entsprechen zwei Strukturen im Reflexionsspektrum: Eine mit zunehmendem $v$ zu kleineren Frequenzen verschobene Plasmakante sowie ein mit zunehmendem $v$ kleiner werdendes Maximum bei der Plasmafrequenz des reinen Metalls. Mit wachsendem Entelektrisierungsfaktor $g$ werden beide Strukturen zu niedrigeren Energien verschoben. Da die experimentell ermittelten Plas- 
maenergien der reinen Metalle bei $2,9 \mathrm{eV}$ für Cs bzw. 3,4 eV für Rb liegen, sollten bei diesen Energien in den Spektren der entsprechenden Suboxide Absorptionsmaxima zu finden sein. In der Tat entsprechen der EO3-Oszillator der Cs-Verbindungen sowie der EO4-Oszillator bei $\mathrm{Rb}_{9} \mathrm{O}_{2}$ den erwarteten Energien. Für $\mathrm{Rb}_{9} \mathrm{O}_{2}$ gibt es keine andere naheliegende Erklärung, dagegen kann der EO3-Oszillator der Cs-Suboxide, wie oben erwähnt, auch mit einem Cs- und/oder einem $\mathrm{O} 2 \mathrm{p}$-Interbandübergang in $\mathrm{Zu}$ sammenhang gebracht werden. Eine Analyse der Intensitätsabhängigkeit von der Sauerstoffkonzentration ist kaum möglich, da die beschriebenen Mechanismen zum Teil gegenläufige Folgen aufweisen.

Eine Polarisationsabhängigkeit kann innerhalb des Löchermodells durch einen anisotropen $g$-Faktor entsprechend einer nichtkugelförmigen Gestalt der Cluster erreicht werden. Der $\mathrm{Cs}_{11} \mathrm{O}_{3}$-Cluster kann durch ein rotationssymmetrisches Ellipsoid angenähert werden mit den Halbachsen $3,5 \AA$ bzw. $2,3 \AA$. Damit ergibt sich $g=0,26$ für eine Orientierung des Lichtvektors in der von den 3 Sauerstoffatomen aufgespannten Ebene und $g=0,48$ für senkrechte Orientierung. Trotz ihrer Neigung gegen die $a c$-Ebene in der Verbindung $\mathrm{Cs}_{11} \mathrm{O}_{3}$ erscheinen die $\mathrm{Cs}_{11} \mathrm{O}_{3}$-Cluster in der Projektion parallel $b$ kreisförmiger (größerer $g$-Wert für Lichtvektor $\| b$ ) als in einer Projektion senkrecht dazu (kleinerer $g$-Wert für Lichtvektor $\perp b$ ). Mit den oben angegebenen $g$ Werten und dem Füllfaktor $v=0,6$ erhält man (ohne Berücksichtigung der Cluster-Neigung) eine gute Anpassung der Spektren für $\mathrm{Cs}_{11} \mathrm{O}_{3}$ im Rahmen des Löchermodells (Abbildung 9). Berechnet

[1] A. Simon, Structure and Bonding 36, 82 (1979).

[2] G. Ebbinghaus u. A. Simon, Chem. Phys. 43, 117 (1980).

[3] W. Bauhofer u. A. Simon, Z. anorg. allg. Chem. 447, 29 (1978).

[4] T. P. Martin, H.-J. Stolz, G. Ebbinghaus u. A. Simon, J. Chem. Phys. 70, 1096 (1979).

[5] M. G. Burt u. V. Heine, J. Phys. C 11,961 (1978).

[6] L. Genzel u. T. P. Martin, Surface Science 34, 33 (1973).

[7] K. Mack u. A. Simon, teilweise wiedergegeben in [1].

[8] A. Simon, Z. anorg. allg. Chem. 395, 301 (1973).

[9] A. Simon, Z. anorg. allg. Chem. 422, 208 (1976).

[10] A. Simon, H.-J. Deiseroth, E. Westerbeck u. B. Hillenkötter, Z. anorg. allg. Chem. 423, 208 (1976).

[11] A. Simon u. H.-J. Deiseroth, Rev. Chim. Minér. 13, $98(1976)$

[12] A. Simon u. E. Westerbeck, Z. anorg. allg. Chem. 428, 187 (1977).
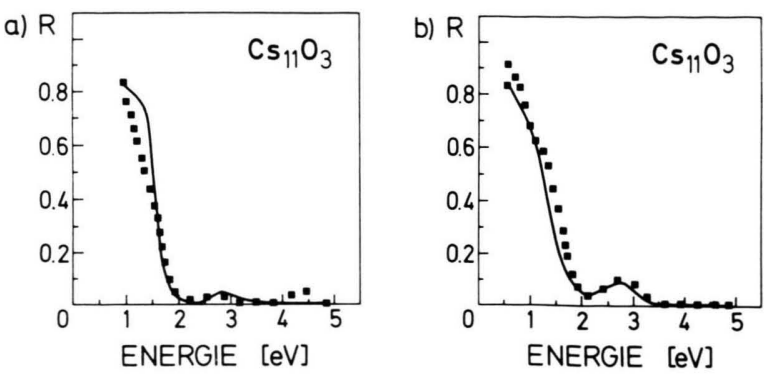

Abb. 9. Anpassung der Reflexionsdaten (schwarze Punkte) von $\mathrm{Cs}_{11} \mathrm{O}_{3}$ nach dem Löchermodell für a) Lichtvektor $b$-Achse, b) Lichtvektor $\perp b$-Achse.

wurde unter Verwendung von (4) die Reflektivität bei senkrechtem Lichteinfall, wobei $\varepsilon=1$ gesetzt und für $\varepsilon_{\mathrm{m}}(\omega)$ die aus der KK-Analyse erhaltenen komplexen Dielektrizitätsfunktionen des reinen Metalls verwendet wurde. Dennoch bleibt die Gültigkeit dieses sehr vereinfachten Modells in seiner Anwendung auf die Alkalimetallsuboxide fraglich, da die Spektren der anderen Suboxide, insbesondere des $\mathrm{Cs}_{7} \mathrm{O}$, nur mangelhaft an dieses Modell angepaßt werden können.

\section{Danksagungen}

Wir danken den Herren Prof. L. Genzel und Dr. T. P. Martin für wertvolle Diskussionen, sowie Herrn F. Kögel für Probenpräparationen. Dem Verband der Chemischen Industrie - Fonds der Chemie - sind wir für die Unterstützung dieser Untersuchungen dankbar.

[13] A. Simon, Z. anorg. allg. Chem. 431, 5 (1977).

[14] H. E. Bennet, M. Silver u. E. J. Ashley, J. Opt. Soc. Amer. 53, 1089 (1963); K. Köhler, private Mitteilung.

[15] G. Metsch, Dissertation Stuttgart 1984.

[16] C. Kunz, Phys. Letters 15, 312 (1965); Z. Physik 196, 311 (1966) - H. Mayer u. St. v. Aufschnaiter, Z. Physik 249, 400 (1972). - H. Mayer u. B. Hietel, Z. Physik 254, 232 (1972). - U. S. Whang, E. T. Arakawa u. T. H. Callcott, Phys. Rev. B 5, 2118 (1972); Phys. Rev. Letters 25, 646 (1979). - N. V. Smith, Phys. Rev. B 2, 2840 (1970). - J. Monin u. G. A. Boutry, Phys. Rev. B 9, 1309 (1974).

[17] R. Hummel, Optische Eigenschaften von Metallen und Legierungen, Springer-Verlag, Berlin 1971.

[IA] I. (i. Endriz u. W. E. Spicer, Phys. Rev. B 4, 4144 (1971); Phys. Rev. B 4, 4159 (1971). 